\title{
Lymphangioms of the women's reproductive system
}

\begin{abstract}
Lymphangiomas are congenital, hamartomatous and uncommon malformations of the lymphatic system that affect the skin and subcutaneous tissues. The most frequently used classification divides these lesions into 2 main groups according to the depth and size of these abnormal lymphatic vessels; arise from kidnappings of lymphatic tissue that should normally communicate with the lymphatic system. Most appear to be developmental lesions that occur relatively early in life, rarely occur in adulthood. The histological features that favor diagnosis are the presence of lymphoid aggregates in the stroma; They appear on any part of the skin and mucous membranes and surgical management with complete surgical excision.
\end{abstract}

Volume II Issue I - 2020

\author{
Vargas-Hernandez Victor Manuel \\ Department of Gynecology, Hospital Juarez de México, México
}

Correspondence: Vargas-Hernandez Victor Manuel, Department of Gynecology, Secretaria de Salud, Hospital Juarez de México, México, Email vvargashernande@yahoo.com.mx

Received: January 09, 2020 | Published: February 17, 2020

Keywords: vascular malformations, genital tract, breast, diagnosis and treatment

\section{Background}

Vascular tumors are rarely found in the female genital tract; Most vascular tumors are incidental findings due to their small size and asymptomatic nature, large lesions are present clinically, with characteristics that mimic gynecological tumors, even in imaging studies. ${ }^{1,2}$ Lymphangiomas are congenital or acquired malformations (secondary to trauma, infections or malignancies) that manifest as a very varied-looking tumor that is formed from embryonic cells in the ducts of the lymphatic system. From 20 to $40 \%$ of cases are associated with chromosomal abnormalities, various aneuploidies or malformations. Lymphangiomas are composed of dilated lymphatic vessels lined by endothelium and more than $50 \%$ of these lesions exist from birth; $90 \%$ appear in the second year of life. These lesions do not spread rapidly, but tend to infiltrate the surrounding tissues and malignant degeneration is very rare. Their size increases after birth and they invade adjacent structures, usually with cavernous spaces upholstered with endothelial and lymphoid cells. From the pathological point of view they are cavities full of lymphatic fluid of three types: capillary, cavernous and cystic (macrocystic with cysts larger than $2 \mathrm{~cm}$ and microcystic otherwise). There are mixed forms and associated with vascular lesions of high flow; They are divided into three histopathological subtypes: capillary (composed of thin capillary walls), cavernous (composed of dilated lymphatic spaces) and cystic (cysts dilated by a layer of endothelium of variable size). Clinically it manifests as: a benign, soft, slow-growing tumor with aesthetic or functional alterations. Its volume is increased by infection or hemorrhage when vascular lesions coexist. They are diagnosed clinically and by imaging studies with ultrasound, computed tomography and magnetic resonance imaging. To define the lesion, its extension and fine needle aspiration must be differentiated from any cyst, lipoma and other vascular malformations. ${ }^{3}$

Differential diagnosis of vascular tumors should be made mainly from endometriotic lesions and melanomas. Immunohistochemistry is useful for differentiating lymphangiomas in difficult cases. (S100, CD31, CD34, CKS, EMA) help in diagnosis. Local excision is suitable for benign vascular lesions. ${ }^{2}$
Lymphangiomas are congenital, hamartomatous and rare and benign malformations of the lymphatic system that affect any part of the skin and mucous membranes. Lymphangiomas can be classified according to the depth and size of abnormal lymphatic vessels. Superficial forms of lymphangioma include circumscribed lymphangioma and acquired lymphangioma, which is also known in the literature as lymphangiectasis. ${ }^{4}$

The most frequently used classification divides these lesions into 2 main groups according to the depth and size of these abnormal lymphatic vessels. Superficial vesicles are called circumscriptum lymphangioma. The deepest group includes cavernous lymphangioma and cystic hygroma. Lymphangiomas can appear on any part of the skin and congenital or acquired mucous membranes. ${ }^{5,6}$

These lesions are soft, vary in size and shape, and tend to grow widely if they are not surgically removed. Typical lesions are multilocular cysts filled with clear or yellow lymphatic fluid.

Lymphangiomas can arise in any part of the body usually grow slowly and patients remain asymptomatic for a long time, and the tumor is identified incidentally during the histopathological examination after excision. It is advisable to remove the lesion with microscopically clear margins. Frequently, diagnosis becomes difficult because it can be confused.

The treatment is not completely standardized, it depends on its size and location; Small lesions, without growth and that do not affect the function of organs are kept under observation. In major lesions, surgical removal is indicated. ${ }^{\text {? }}$

\section{Epidemiology}

It represents approximately 1 to $4 \%$ of all vascular malformations and $26 \%$ of all benign vascular tumors; The remaining $5 \%$ can be distributed throughout the rest of the body. 8 . No racial predominance is reported for lymphangiomas. Sex equality for lymphangiomas can become apparent at any age, but the highest incidence occurs at birth or early in life. About $50 \%$ of lymphangiomas are seen at birth, and most lymphangiomas are evident when the patient is 5 years old. It can also be documented in fetuses 9. There is no racial or gender 
predilection. Lymphangiomas usually occur at birth or in the first years of life, while the acquired form. It often occurs in adulthood. ${ }^{9,10}$

\section{Clinical evaluation}

Clinically it appears as multiple vesicular papules, grouped or scattered, translucent or hemorrhagic lesions consisting of a combination of blood and lymphatic elements, you can see scattered purple areas within the vesicles shaped papules; In the acquired form, there is often coexisting lymphedema. Associated symptoms may include pruritus, pain, burning, lymphatic drainage, infection and aesthetic problems; they are lesions ${ }^{9}$ they are soft lesions, with different sizes and shapes, and they will generally grow if they are not removed. In most cases, a clinical diagnosis can be made based on the history and examination. As necessary, the biopsy to confirm the diagnosis and images to assess the depth and extent of a lesion. The sedimentation of blood that separates the cellular components of the bottom and the serum from the top of the lagoons. ${ }^{11}$ The typical history involves a small amount of vesicles in the skin at birth or shortly thereafter. In the following years, they tend to increase in number, and the area of the skin involved continues to expand. Vesicles or other skin abnormalities may not be noticed until several years after birth. In general, the lesions are asymptomatic, but, occasionally, patients may have spontaneous episodes of minor bleeding and abundant drainage of clear fluid from broken vesicles. Lymphangiomas can affect several sites. ${ }^{9,12}$ They are associated with minor bleeding, recurrent cellulitis and loss of lymphatic fluid. Genital lymphangiomas can be complicated by cellulite. ${ }^{5}$ Magnetic resonance imaging can help define the degree of involvement and the complete anatomy of lymphangioma lesion help prevent unnecessary extensive and incomplete surgical resection, due to the association with a high rate of recurrence.

\section{Pathological aspects of lymphangiomas}

The lesions consist of a collection of large lymphatic cisterns that communicate through dilated dermal lymphatic channels lined with endothelial cells. The overlying epidermis is usually a clot or hyperkeratotic and has an irregular lengthening of the rete pegs. There are no atypical vascular characteristics, nuclear atypia, mitotic activity or koilocytic changes. A mild to moderate inflammatory infiltrate may be present large irregular vascular spaces lined with a single layer of flattened endothelial cells within a fibroblastic stroma or collagen. ${ }^{13}$

Histological findings microscopically, the vesicles in the circumscriptum lymphangioma are very dilated lymphatic channels that cause the papillary dermis to expand. They may be associated with acanthosis and hyperkeratosis. These channels are numerous in the upper dermis and often extend to the subcutis. The nodules in the cavernous lymphangioma are characterized by large and irregular channels in the reticular dermis and subcutaneous tissue that are lined by a single layer of endothelial cells. The surrounding stroma consists in loose or fibrotic connective tissue with several inflammatory cells. ${ }^{14}$

\section{Gynecological lymphangioms}

Lymphangioma is a rare, non-epithelial and benign disease of the vulva, it can be classified according to its origin as primary or more frequently as secondary (or acquired). The clinical presentation can also be asymptomatic, the patient's main complaints included vulvar pain, difficulty performing daily tasks and discomfort during sexual intercourse; with great tumor formation that consisted of multiple small confluent vesicles that replaced the normal vulvar labial anatomy from the clitoris, predominantly on the right side. Hypertrophy of the labia minora. Primary vulvar lymphangioma usually develops after birth and during early childhood, it can occur at any age, even in older patients. Congenital lesions usually develop as the patient grows and their external genitals evolve, until the lesions eventually become clinically evident during adulthood or at any age. Due to its clinical appearance and size, it often results in aesthetic and psychosexual disorders; It is a pseudo benign tumor, treatment is recommended for symptomatic relief, as well as for the improvement of aesthetic or psychological problems and discomfort. The psychosexual implications of this diagnosis are significant and cause great anxiety. Carvenous lymphangioma is an extremely rare benign tumor of the vulva as a soft mass, vulvar circumscriptum lymphangioma typically presents as multiple macroscopic vesicles of various sizes, it may be impossible to clinically distinguish vulvar circumscriptum lymphangioma from other vulvoperineal skin diseases. High-grade squamous intraepithelial cannot be grossly distinguished from the circumscribed vulvar lymphangioma because the multiple and warty vesicles that constitute the characteristic macroscopic appearance of the vulvar lymphangioma circumscribe hinder their distinction. In this sense, our cases of high-grade squamous intraepithelial lesion, located adjacent to the vulvar lymphangioma circumscribed, support the notion that active surgical excision is necessary for the treatment of vulvar lymphangioma circumscribed. ${ }^{15}$ At present, there is no consensus About the standard treatment. Treatment modalities include surgical excision, abrasive methods (carbon dioxide laser, liquid nitrogen, electrocoagulation or sclerosing therapy) and observation; It has not been shown that local therapy reliably improves the patient's symptoms, such as pain and/or pruritus. Surgical treatment options can be considered favorably when the patient meets the following criteria:

1) A large mass and deep lesions of vulvar LC

2) The presence of distressing symptoms, such as pain, pruritus, edema, secretion and secondary infection

3) Non-surgical treatment failure. ${ }^{16}$

The treatment option is complete surgical resection with a cosmetic approach and laser vaporization with $\mathrm{CO}_{2}$ has similar success and recurrence rates. Conservative management (observation) is a valid option if the patient is asymptomatic. There is currently no established standard of care. The recurrence of the lesion is frequent, regardless of the choice of treatment. It is known that local excision as a high recurrence rate and treatment failure. Sclerotherapy and radiation. Accompanying physiotherapy is generally recommended to improve edema. The selection of treatment generally depends on the location and size of the lesions. Observation is an option in asymptomatic patients who refuse treatment. With respect to local excision, a recurrence rate of $23.1 \%$ has been reported between 6 and 81 months after treatment. The recurrence rate seems to be higher when the initial lesion is greater than $7 \mathrm{~cm}$, despite radical excision. ${ }^{8,16-19}$ Vaginal lymphangioma is an extremely rare lesion, arising from the left lateral wall, to alert gynecologists of the need to consider lymphangioma in their differential diagnosis. After diagnosis, we recommend a surgical approach to management, it is a benign tumor. Its clinical manifestations can be confused with malignant tumors or other benign tumors. Colposcopy and vaginal biopsy are two useful diagnostic tools that can help make an accurate diagnosis of the lesion and ensure proper treatment. Ultrasound and magnetic resonance imaging had limited utility in the evaluation of this superficial, sensitive and well circumscribed lesion. Lymphangioma is characterized by groups of 
thin-walled vessels that are filled with a transparent liquid. Epithelial hyperplasia and hyperkeratosis give rise to firm lesions, can be congenital or acquired. Acquired cases are seen mainly after radiation therapy to the pelvis, cases are diagnosed to exclude metastatic deposits. Excision biopsies are diagnostic; The preferred treatment is surgical excision. Clitoral lymphangioma is very rare and the removal resulted in a good prognosis..$^{20-22}$

Lymphangioma of the uterine tuba is extremely rare alone, dilations of the preexisting canals do not take the form of circumscribed nodular tumors and occur only when there is a physical one, that is, the accumulation of secretions or the removal of foreign matter, which it does not require static conditions in the cases that accompanied the tubas in all other normal exams. ${ }^{23}$ Ovarian lymphangioma is extremely rare and a pathological examination is necessary to arrive at the correct diagnosis; where the ovaries have a rich vascular supply and it has been postulated that the rarity of this tumor is due to cyclic changes that the ovary undergoes during the reproductive years. The contents in the cystic spaces, the characteristic morphology, with lymphocytic infiltrates and immunohistochemistry helps diagnosis in difficult cases; They usually grow slowly and patients remain asymptomatic for a long time, and the tumor is identified incidentally during the histopathological examination after excision. It is advisable to remove the lesion with microscopically clear margins. Frequently, the diagnosis becomes difficult because it can be confused remain asymptomatic for a long time, and the tumor is identified incidentally during the histopathological examination after excision. With a malignant ovarian mass, especially in postmenopausal women. Should be included in the differential diagnosis of an ovarian cystic mass, ultrasound revealed a right adnexal mass with solid and cystic components.

Immunohistochemistry (D2-40) confirmed the diagnosis of ovarian lymphangioma that coexists with mature cystic teratoma; Laparoscopic excision can be the ${ }^{24-26}$ treatment method. Giant uterine lymphangioma, a rapidly growing abdominal tumor and its accompanying symptoms, progressive abdominal distension, lumbago and developed edema of the legs, are rare benign tumors of the lymphatic system that are usually congenital and is an asymptomatic cystic mass. The diagnosis is made in the postoperative period; ultrasound and magnetic resonance are useful; No adverse outcomes were reported during pregnancy. Surgical resection is usually necessary to confirm the diagnosis of lymphangioma. ${ }^{27,28}$

\section{Lymphangiom of the breast}

The lymphangioma of the breast is a rare entity in the breast with only a few cases reported so far. Lymphangiomas generally consist of dilated lymphatic channels covered by endothelium. This may be due to blockage of lymphatic channels with secondary dilation, congenital weakness of the lymphatic wall or proliferation of lymphatic vessels. Subsequently, these lesions are enlarged over time by the accumulation of fluid. These cystic spaces in turn communicate with large dermal lymphatic vessels and subepidermal vesicles, but not with the regional lymphatic system, they occur in the upper outer quadrant, Spence's tail or in the subareolar space. Differential diagnoses to consider include simple cysts and fibrocystic disease, lymphocele, hematoma and hemangioma. The images are an integral part to reach a diagnosis. Ultrasound is useful in young women with dense breasts and helps differentiate solid masses from cystic masses. Magnetic resonance facilitates the evaluation of the depth and extent of the tumor, which helps therapy. The confirmatory diagnosis of lymphangioma is made on the basis of histopathology. Lymphangiomas are treated primarily for aesthetic and functional reasons. Lack of treatment can lead to secondary complications, such as infection and bleeding. Malignant degeneration in squamous cell carcinoma has been reported in longterm cases.

The treatment of choice depends on the depth and location of the lesion along with the age of the patient and the general state of health. Wide surgical excision of the cisterns facilitated by MRI is the most effective treatment modality and remains the standard of care. Steroid injections and sclerosing agents cause tissue sclerosis and decrease the size of the lesion. The carbon dioxide laser can produce functional and cosmetically acceptable results by vaporizing some of the surface lymphatic vessels. ${ }^{14}$ Streptococcal lysine (OK-432), a biological response modifier, induces local inflammatory cytokines that increase the permeability of endothelial cells, lymphatic drainage and flow that lead to the contraction of cystic spaces, but all of these are associated with a high recurrence 16tases and, in addition, subsequent surgery becomes technically difficult due to sclerosed tissue. Therefore, they are used as adjuvants for surgery. For poor surgical candidates, the option considered is radiotherapy. ${ }^{29}$ The definitive diagnosis is based on clinical, imaging and histopathological findings.

Histologically, numerous small spaces are identified that contain eosinophilic amorphous fluid and some lymphocytes and are surrounded by a monolayer of diminished endothelial cells. Some lymphatic channels may contain blood from secondary hemorrhage, large collections of lymphoid cells in the stroma, sometimes with lymphoid follicle formation and irregularity confirm the diagnosis of lymphangioma. ${ }^{3}$ It is believed that they come from malformations resulting from hyperproliferation of the vessels lymphatic, weakness in the lymphatic walls or other obstruction of lymphatic drainage are usually painless. Therefore, most are treated cosmetically through the use of imaging, it has been suggested that MRI is the most informative, since lymphangiomas generally show low T1 intensity and high intensity in T2 images. In addition, there is an improvement of the septa, with tumors that often appear multisected, the standard reference for diagnosis is performed by excisional biopsy, for patients in whom surgery is contraindicated, a guided percutaneous sclerosis can be performed. ${ }^{29-33}$

\section{Lingangioma management}

Superficial and superficial lymphangiomas can be difficult to treat the treatment of choice for any type of lymphangioma is still surgical excision. Wide local excision of the affected lymphatic channels is necessary since recurrence is common. It has been reported that recurrence rates are as high as $23 \%$ higher for small and superficial lymphangiomas. Destructive treatments with carbon dioxide $\left(\mathrm{CO}_{2}\right)$ laser, Nd-YAG long pulse laser and electrosurgery improve symptoms. Cryotherapy, superficial radiotherapy and sclerotherapy with hypertonic saline solution at $23.4 \%$ are less used modalities. Direct injection of a sclerosing agent, which includes $1 \%$ or $3 \%$ sodium tetradecyl sulfate, doxycycline or ethanol, can become lymphatic malformations. Compression can reduce the swelling caused by lymphedema. Infection prevention is crucial. Lymphangioma does not respond to radiotherapy or steroids. However, propranolol represents a potential option, which can be beneficial even for intractable diffuse lymphangiomatosis. Sodium tetradecyl sulfate can be used with care for treatment not indicated in circumscriptum lymphangioma. ${ }^{34}$ Antibiotics are given for secondary cellulite. 
The preferred treatment for lymphangiomas is complete surgical excision. Local recurrences are common in lymphangiomas. Adequate excision of lymphangiomas can be difficult and sometimes unfeasible. This problem is the main reason for the high recurrence rate..$^{35,36}$ Sclerotherapy with bleomycin and postoperative vacuum assisted closure devices may reduce the risk of recurrence and infection.., 37

\section{Clinical follow-up}

Patients with lymphangiomas should be monitored and treated for cellulite, especially those with broken vesicles, which provide an entry portal for infection. The most common complications associated with circumscriptum lymphangioma include rare cellulitis and leakage of lymphatic fluid from squamous cell carcinoma, verruciform xanthoma and lymphangiosarcoma. Acquired lymphangiomas are not believed to have malignant potential; The associated chronic lymphedema puts the patient at risk of lymphangiosarcoma, cutaneous angiosarcoma in massive lymphedema located in morbidly obese patients. Acquired lymphangiectasia can be painful and poor lymphatic drainage can cause a bacterial infection. Lymphangiomas are benign lesions that are difficult to diagnose and manage. These lesions are best managed by an interprofessional team the treatment of choice is surgical excision recurrence rates greater than $30 \%$. Surgery may be associated with complications such as lymphatic leaks, fistula formation and chronic wounds. 5

\section{Conclusion}

Benign vascular tumors such as lymphangiomas present clinically, simulating common gynecological tumors; some are asymptomatic and are incidentally found, are difficult to distinguish clinically and imaging from other neoplasms, the biopsy is the standard reference and its treatment is complete surgical resection.

\section{Acknowledgments}

None.

\section{Funding}

None.

\section{Conflicts of interest}

The authors declare there are no conflicts of interest.

\section{References}

1. Uma S Andola, Sainath K Andola. Vascular tumours of the female genital tract: a clinicopathologic study of 11 cases. Journal of Clinical and Diagnostic Research. 2011;5(6):1241-1246.

2. Kondi-Pafiti A, Kairi-Vassilatou E, Spanidou-Carvouni H, et al. Vascular tumors of the female genital tract: a clinicopathological study of nine cases. Eur J Gynaecol Oncol. 2003;24(1):48-50.

3. Vargas-Hernández VM, Tovar-Rodríguez JM, Moreno-Eutimio MA, et al. Giant cystic lymphangioma of the breast. Case report with 20-year follow-up and literature review. Cir Cir. 2014;82:81-86.

4. Noia G, Maltese PE, Zampino G, et al. Cystic hygroma: a preliminary genetic study and a short review from the literature. Lymphat Res Biol. 2019;17(1):30-39.

5. Robert A Schwartz, Dirk M Elston. Lymphangioma. Medscape, 2019.

6. Kolay SK, Parwani R, Wanjari S, et al. Oral lymphangiomas - clinical and histopathological relations: an immunohistochemically analyzed case series of varied clinical presentations. J Oral Maxillofac Pathol. 2018;22 (Suppl 1):S108-S111.

7. Michael Piernick D, Syed Hamzah Mahmood, Steven Daveluy. Acquired lymphangioma circumscriptum of the genitals in an individual with chronic hidradenitis suppurativa. JAAD Case Rep. 2018;4(1):64-66.

8. Sluga MC, Domenech MM, Peremateu MS, et al. Report of 4 cases of vulvar lymphangioma: an update. Obstet Gynecol Int J. 2018;9(2):143146.

9. Ersoy AO, Oztas E, Saridogan E, et al. An unusual origin of fetal lymphangioma filling right axilla. J Clin Diagn Res. 2016;10(3):QD09QD11.

10. Chang MB, Newman CC, Davis MD, et al. Acquired lymphangiectasia (lymphangioma circumscriptum) of the vulva: Clinicopathologic study of 11 patients from a single institution and 67 from the literature. Int $J$ Dermatol. 2016;55(9):e482-e487.

11. Sehgal VN, Sharma S, Chatterjee K, et al. Unilateral, blaschkoid, large lymphangioma circumscriptum: micro- and macrocystic manifestations. Skinmed. 2018;16(6):411-413.

12. Zaballos P, Del Pozo LJ, Argenziano G, et al. Dermoscopy of lymphangioma circumscriptum: A morphological study of 45 cases. Australas J Dermatol. 2018;59(3):e189-e193.

13. Saccone G, Di Meglio L, Di Meglio L, et al. Prenatal ultrasound diagnosis of fetal chest wall cystic lymphangioma: an Italian case series. Eur J Obstet Gynecol Reprod Biol. 2019;236:139-142.

14. Hara H, Mihara M, Anan T, et al. Pathological investigation of acquired lymphangiectasia accompanied by lower limb lymphedema: lymphocyte infiltration in the dermis and epidermis. Lymphat Res Biol. 2016;14(3):172-180

15. Jha AK, Lallas A, Sonthalia S. Dermoscopy of cutaneous lymphangioma circumscriptum. Dermatol Pract Concept. 2017;7(2):37-38.

16. Bae GE, Yoon G, Song YJ, et al. High-grade squamous intraepithelial lesion arising adjacent to vulvar lymphangioma circumscriptum: a tertiary institutional experience. Oncotarget. 2016;7:48120-48129.

17. Yoon G, Kim HS, Lee YY, et al. Clinical outcomes of primary surgical treatment for acquired vulvar lymphangioma circumscriptum. Arch Gynecol Obstet. 2016;293:157-162.

18. Liu XY, Zhang S, Heng Z, et al. Lymphangioma circumscriptum in vulva with klippel-trenaunay syndrome. Chin Med J. 2018;131(4):490-491.

19. Yoon KR, Mo AJ, Park SH, et al. Carvenous lymphangioma of the vulva. Obstet Gynecol Sci. 2015;58(1):77-79.

20. Yoon G, Kim HS, Lee YY, et al. Clinical outcomes of primary surgical treatment for acquired vulvar lymphangioma circumscriptum. Arch Gynecol Obstet. 2016;293:157-162.

21. Park SI, Hong KP, Lee KW, et al. A case of lymphangioma of the clitoris. Korean J Gynecol Oncol Colposc. 2003;14(3):239-241.

22. Tomai XH, Phan TH, Metrics PX. Lymphangioma of the vagina. JOGC. 2013:35(9):827-830

23. Kesterson JP, Lele SB. Tumors of the vulva and vagina. In: Raghavan D, Blanke CD, Johnson DH, editors. Textbook of uncommon cancer. 4th ed. Hoboken, New Jersey: John Willey \& Sons; 2012.

24. Lawrence W. Strong, Lymphangioma of the fallopian tube. AJOG. $1925 ; 10(6): 853-855$.

25. Uma S Andola, Sainath K Andola. Vascular tumours of the female genital tract: a clinicopathologic study of 11 cases. Journal of Clinical and Diagnostic Research. 2011;5(6):1241-1246. 
26. Naik SA. Rare case of ovarian cystic lymphangioma managed at laparoscopy. J Gynecol Endosc Surg. 2011;2(2):97-100.

27. Vaidya KM, Shrestha B. Ovarian lymphangioma with mature cystic teratoma. J Nepal Health Res Counc. 2019;17(1):128-130.

28. Tatsuro F, Tatsuro F, Tatsuro F, et al. Cavernous lymphangioma arising from uterine corpus. Ginec Oncol. 2003;90(1):195-199.

29. Shore EM, Latta E, Colak E, et al. Uterine lymphangioma in pregnancy. Obstet Gynecol. 2014;124(2):472-475.

30. Bharati H, Vineet B. Lymphangioma of the breast. BMJ Case Rep. 2014;2014:bcr2014203937.

31. Rusdianto E, Murray M, Davis J, et al. Adult cystic lymphangioma in the inner quadrant of the breast-rare location for a rare disease: a case report. Int J Surg Case Rep. 2016;20:123-126.
32. Daniel R Principe, Jonathan Rubin, Andreea Raicu, et al. Massive adult cystic lymphangioma of the breast. J Surg Case Rep. 2019;(2):rjz027.

33. Principe DR, Jonathan R, Andreea R, et al. Massive adult cystic lymphangioma of the breast. J Surg Case Rep. 2019;2:1-3.

34. Park T, Lee HS, Jung EJ, et al. Concomitant breast and axillary lymphangioma in an adult. A case report and a review of the literatura. Medicine. 2018;97(45):e12946.

35. Jenkinson HA, Wilmas KM, Silapunt S. Sodium tetradecyl sulfate: a review of clinical uses. Dermatol Surg. 2017;43(11):1313-1320.

36. Ekelem C, Thomas L, Van Hal M, et al. Radiofrequency therapy and noncosmetic cutaneous conditions. Dermatol Surg. 2019;45(7):908-930.

37. Porwal PK, Dubey KP, Morey A, et al. Bleomycin sclerotherapy in lymphangiomas of head and neck: prospective study of 8 cases. Indian $J$ Otolaryngol Head Neck Surg. 2018;70(1):145-148. 